\title{
A new three-dimensional chaotic flow with one stable equilibrium: dynamical properties and complexity analysis
}

https://doi.org/10.1515/phys-2018-0037

Received February 1, 2018; accepted March 5, 2018

\begin{abstract}
This paper proposes a new three-dimensional chaotic flow with one stable equilibrium. Dynamical properties of this system are investigated. The system has a chaotic attractor coexisting with a stable equilibrium. Thus the chaotic attractor is hidden. Basin of attractions shows the tangle of different attractors. Also, some complexity measures of the system such as Lyapunov exponent and entropy will are analyzed. We show that the Kolmogorov-Sinai Entropy shows more accurate results in comparison with Shanon Entropy.
\end{abstract}

Keywords: Chaotic flow, stable equilibrium, hidden attractor, basin of attraction, entropy, complexity

PACS: 05.45.Ac, 05.45.Pq

\section{Introduction}

Chaotic systems and their dynamical properties are interesting topics in nonlinear dynamics. Small differences in initial conditions (such as those due to rounding errors in numerical computation) yield widely diverging outcomes for such dynamical systems, rendering long-term prediction of their behavior impossible in general. This happens even though these systems are deterministic, meaning that their future behavior is fully determined by their initial conditions, with no random elements involved. In other words, the deterministic nature of these systems does not make them predictable. This behavior is known as deterministic chaos, or simply chaos [1-3].

For many years, researchers have believed that a chaotic attractor is related to a saddle equilibrium [4-7]. However it is clear now that the existence of a saddle point is not a necessary condition for existence of chaotic solutions. The reason is that recently many new chaotic systems have been proposed without any equilibria [8], with one stable equilibria [9], with a line of equilibria [10,11], with a curve of equilibria [12,13] and with a plane of equilibria [14]. It seems the relation between equilibria and their stable and unstable manifolds is unknown to us. From a point of view, attractors of dynamical systems have been categorized into two groups. The first one is hidden attractor. An attractor is called hidden if its basin of attraction does not intersect with a small neighborhood of any equilibrium point [15-18]. The second one is self-excited attractor. An attractor is called self-excited if its basin of attraction is associated with an unstable equilibrium [1921]. In the case of chaotic attractors in systems with a stable equilibrium, at least there are two attractors simultaneously (strange attractor and stable equilibrium attractor). These attractors are called multi-stable since the final state of the system is dependent on its initial condition [22-25].

There are many features which measure the complexity of dynamical systems. Features such as Lyapunov exponents, Entropy, fractal dimension and correlation dimen- 
sion $[3,26]$ are some examples of them. Also, these measures quantify chaotic attractors [3]. Entropy measure is the best when only a short time series of system is available [27]. In this paper, we propose a new three-dimensional chaotic flow with only one stable equilibrium. In this case the strange attractor is hidden, since the existence of an unstable equilibrium in its basin is impossible.

It should be noted that while there are many chaotic flows in the literature, only a little part of them are systems with hidden attractors. In that little part, only a few of them are systems with stable equilibria only. Another important aspect about our new proposed system is that it is multi-stable. Multistability is an important topic in nonlinear dynamics and chaos [28-33]. In some occasions multistability is unwanted, while in some cases it is desired. When it is important in a dynamical system to work in a specific state and not go out from it, then multistability in that system is a potential danger (because due to any disturbance the system can go to a new unwanted situation). On the other hand, multistability makes systems flexible without tuning parameters [34]. In any case, better understanding of such systems may be of interest.

Chaotic systems with hidden attractors and multistability are very important in engineering and can be much challenging in applications like control [35,36], synchronization [37-42], anti-synchronization [43], image encryption [44,45], and so on.

The aim of this paper is to

a) enrich the list of known chaotic systems with hidden attractors, especially systems with stable equilibrium (which are the rarest kind),

b) enrich the list of known chaotic systems with multistability and a better analysis of them by the help of looking at their basins of attraction,

c) comparing complexity measurement tools in order to determine better indexes when we deal with real timeseries instead of systems' equations.

The rest of the paper is organized as follows: the system's attractors and their basins of attraction are discussed in Section 2. In Section 3 we do the bifurcation analysis for the proposed system. Entropy analysis of the system is investigated in Section 4. Finally, Section 5 concludes the paper.

\section{System description}

Consider the following general form of a threedimensional chaotic flow,

$$
\begin{aligned}
& \dot{x}=z \\
& \dot{y}=-x-z \\
& \dot{z}=f(x, y, z)
\end{aligned}
$$

This structure is inspired from system $S E_{14}$ in Ref. [46]. Using trial and error by the help of computer, we tried to find a simple choice for $f(x, y, z)$ with the following conditions:

a) The equation $f(0, y, 0)=0$, should have only one solution, say $y^{\star}$, which means the system has only one equilibrium in $\left(0, y^{\star}, 0\right)$.

b) That equilibrium must have only eigenvalues with negative real parts (which means it should be stable).

c) The system should have chaotic solution for some initial conditions.

We used a simple, but time consuming method. We produced random sets of parameters to find bounded oscillatory solutions. Then for the most elegant [47] results, we fixed all parameters but one, and changed that one manually seeking for the largest positive Lyapunov exponent. Finding the first chaotic solution, we tuned carefully all other parameters, trying to find more elegant values without losing the chaotic solution.

The simplest case we found is:

$$
\begin{aligned}
& \dot{x}=z \\
& \dot{y}=-x-z \\
& \dot{z}=-1.2 x+4 y-z+z^{2}+2 x y+b y z
\end{aligned}
$$

where $x, y$ and $z$ are variables and $b$ is the system's parameter. By setting zeros the right hand side of Eq. (2), the equilibria of the system can be obtained by Eq. (3)

$$
\begin{aligned}
& z=0 \\
& x=0 \\
& y=0
\end{aligned}
$$

So the system has an equilibrium point in the origin. In order to analyze its stability, we calculate the Jacobian matrix of the system in origin,

$$
J=\left[\begin{array}{ccc}
0 & 0 & 1 \\
-1 & 0 & -1 \\
-1.2 & 4 & -1
\end{array}\right]
$$

By solving $|J-\lambda I|=0$, characteristic equation is obtained by

$$
\lambda^{3}+\lambda^{2}+5.2 \lambda+4=0
$$



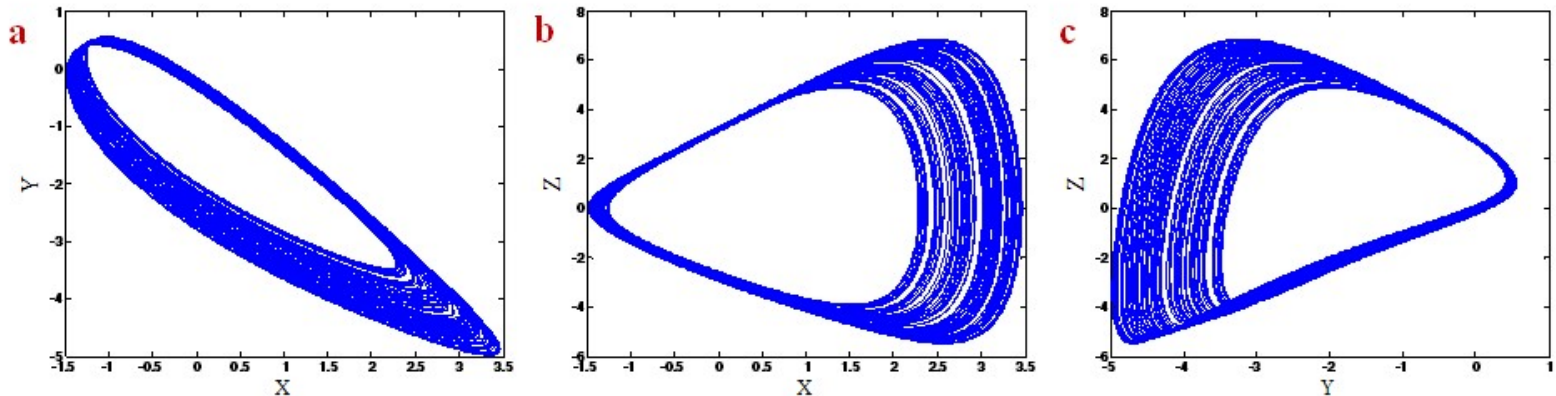

Figure 1: Three projections of strange attractor of System (2) with initial conditions $(3.95,-3.91,3.17) a)$ in $x-y$ plane, b) in x-z plane, c) in y-z plane.

Thus, the Eigenvalues are $\lambda_{1}=-0.7942, \lambda_{2,3}=$ $-0.1029 \pm 2.2419 i$. It means that the origin is stable (spiral node, since it has three eigenvalues with negative real parts, and a pair of them is complex).

System (2) has a chaotic attractor for $b=0.4$ (for e.g. initial conditions $(3.95,-3.91,3.17)$. Figure 1 shows three projections of this strange attractor. The system has only one equilibrium, which is stable. Thus, the strange attractor is hidden. Figure 2 shows basin of attraction of System (2) in the plane $z=0$ for intervals $x \in[-5,40]$, $y \in[-20,5]$. The red region in the figure shows initial conditions, which lead to the stable equilibrium. Initial conditions in the cyan region lead to chaotic attractor and initial conditions in the yellow region lead to unbounded orbits. Black point in the figure is the origin (which is the stable equilibrium). As the figure indicates the strange attractors of the system cannot be found with initial conditions near the origin. Therefore, strange attractor of the system is hidden.

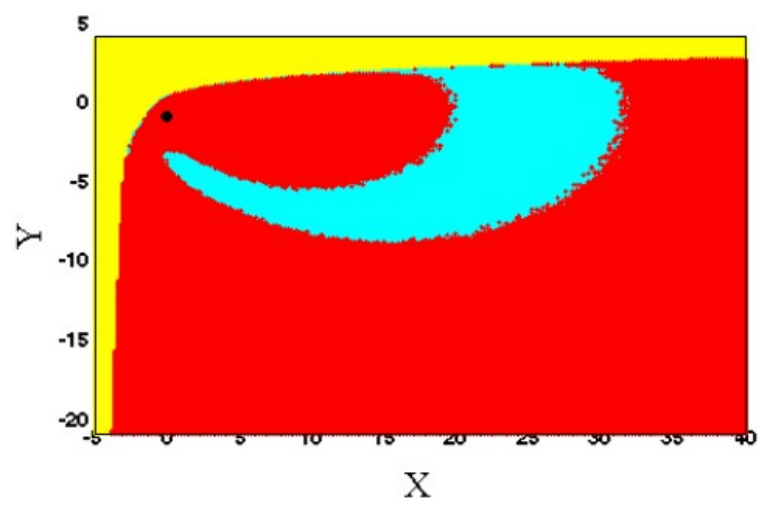

Figure 2: Basin of attraction of System (2) for $b=0.4$. Initial conditions in the red region lead to stable equilibria, initial conditions in the cyan region lead to strange attractor and initial conditions in the yellow region lead to unbounded orbits.

\section{Bifurcation analysis}

Bifurcation diagram is a helpful tool, which can exhibit different dynamical solutions of a system. Figure 3 shows bifurcation diagram of System (2) with respect to changing parameter $b$. The figure depicts that the system has an inverse period doubling route to chaos by increasing parameter $b$. Also the system has a jump from a limit cycle to its stable equilibria in $b=0.424$. Lyapunov exponents diagram in Figure 4 proves different dynamics that can be seen in the bifurcation diagram. Also the jump from limit cycle to the fixed point can be detected in Lyapunov exponents diagram.

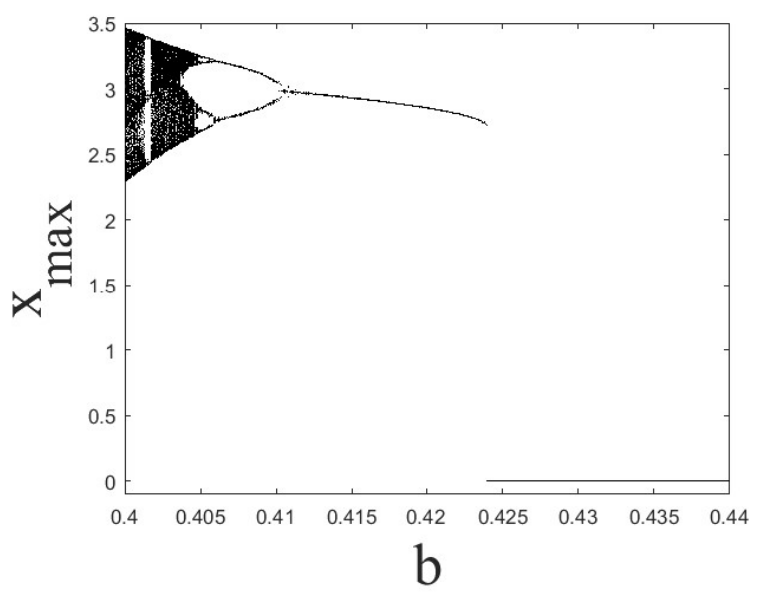

Figure 3: Bifurcation diagram of System (2) with respect to changing parameter $b$ in the interval $[0.4,0.44]$ and forward continuation. 


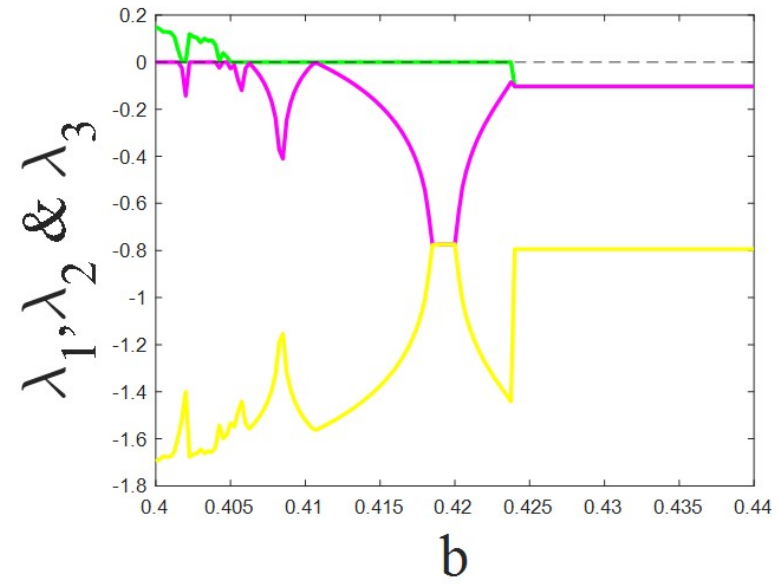

Figure 4: Three Lyapunov exponents of System (2) with respect to changing parameter $b$ in the interval $[0.4,0.44]$.

\section{Entropy analysis}

Entropy is a measure of unpredictability [48]. For the first time, Boltzmann defined it mathematically. Then Shannon defined a more general way for measuring the entropy [49]. Its definition is as follows,

$$
H=-\sum_{i} \rho_{i} \log \left(\rho_{i}\right)
$$

where $\rho_{i}$ is the probability of every possible $i$ states. As bifurcation diagram of Figure 3 shows, the system has an inverse period doubling route to chaos by increasing parameter $b$. So, the system has more complex dynamic in lower values of parameter $b$ and more simple dynamic in larger values. Figure 5 shows entropy of System (2) with respect to changing parameter $b$. The entropy is calculated using Eq. (6) and the Poincare section of the system's time series (peaks of $x$ ) in each parameter $b$. The figure shows that the complexity of dynamic is decreased by increasing parameter $b$ and the dynamic becomes more predictable. Also, the system shows decreasing in the complexity of the periodic window near the parameter $b=0.402$ (as can be seen in the Lyapunov exponent).

In non-periodic attractors, there are infinite number of states in partitions of phase space. So, it is pointless to study the entropy of Eq. (6). In other words, the entropy should be measured relative to another trajectory and per unit of time [27]. Therefore, a new entropy measure is defined as Eq. (7). The entropy is defined using the first Poincaré recurrence times (FPRs) denoted by $\tau_{i}$,

$$
H_{K S}(\beta[\varepsilon])=\frac{1}{\tau_{\min }(\beta[\varepsilon])} \sum_{\tau} \rho(\tau, \beta[\varepsilon]) \log \left(\frac{1}{\rho(\tau, \beta[\varepsilon])}\right)
$$

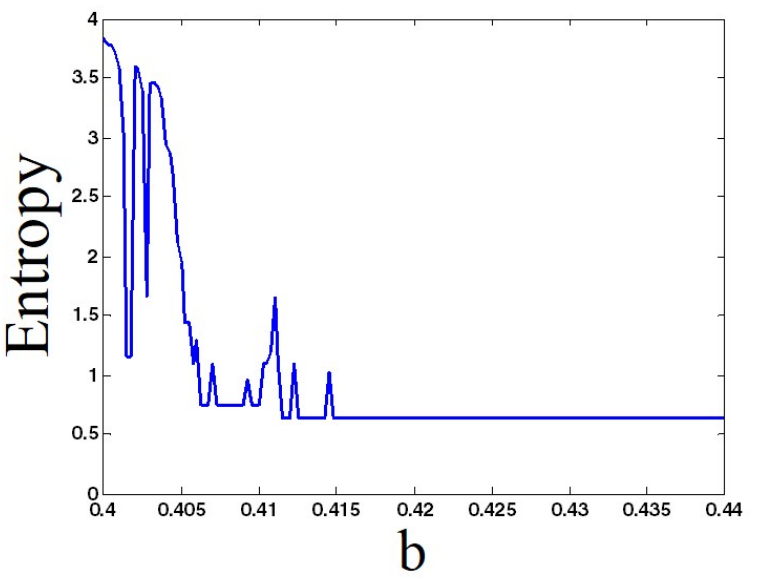

Figure 5: Entropy of System (2) with respect to changing parameter $b$. It is calculated using peaks of $x$ and run time $=20000$. Initial conditions are selected with forward continuation.

$\beta$ is a $\mathrm{D}$-dimensional box in the phase space with side $\varepsilon_{1}$ where the FPRs are observed. $\rho(\tau, \beta)$ is the probability distribution of $\tau_{i}$. The entropy is called Kolmogorov-Sinai $[27,50]$. It has been proved that for a smooth chaotic system $H_{K S}$ is equal to the sum of all positive Lyapunov exponents [51, 52]. Figure 6 shows the Kolmogorov-Sinai entropy with respect to changing parameter $b$. This entropy shows a more proper view about complexity of the system. It depicts that in high values of parameter $b$ there are no positive Lyapunov exponents and so the Entropy is zero. Also, it shows a peak in the bifurcation point from period two to period one. Also decreasing the complexity of system in the inverse route of period doubling can be seen in Figure 5.

\section{Conclusion}

In this paper a new three-dimensional chaotic flow has been proposed. The system had a stable equilibrium point. Thus, its chaotic attractor was hidden. Dynamical analysis of the system has shown an inverse period doubling route to chaos with respect to increasing its parameter. Positive Lyapunov exponent has proved the presence of chaos in this dynamical system. Entropy analysis of the system was done to investigate the predictability of the dynamics using only its time series. We showed that the KolmogorovSinai entropy shows more accurate results in comparison with Shanon entropy. 


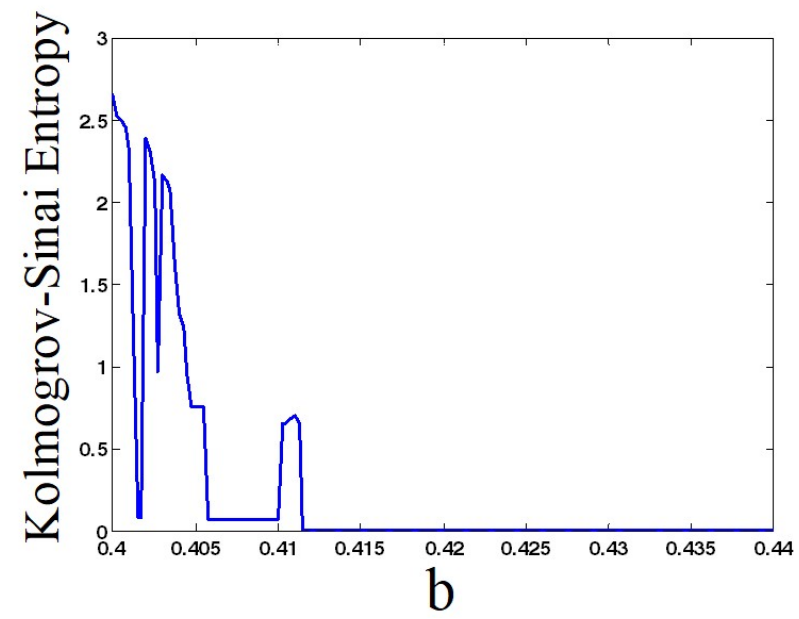

Figure 6: Kolmogorov-Sinai Entropy of System (2) with respect to changing parameter $b$. It is calculated using peaks of $x$ and run $_{\text {time }}=20000$. Initial conditions are selected with forward continuation.

Acknowledgement: TK has been supported by the Polish National Science Centre, MAESTRO Programme-Project No. 2013/327 08/A/ST8/00/780.

\section{References}

[1] Werndl C., What are the new implications of chaos for unpredictability?, Brit. J. Phil. Sci., 2009, 60(1), 195-220.

[2] Chen G., Ueta T., Chaos in circuits and systems, World Scientific, Singapore, 2002.

[3] Hilborn R.C., Chaos and nonlinear dynamics: an introduction for scientists and engineers, Oxford University Press Oxford, 2000.

[4] Lorenz E.N., Deterministic nonperiodic flow, J. Atmospheric Sci., 1963, 20(2), 130-141.

[5] Rössler O.E., An equation for continuous chaos, Phys. Lett. A, 1976, 57(5), 397-398.

[6] Chen G., Ueta T., Yet another chaotic attractor, Int. J. Bifurcat. Chaos, 1999, 9(07), 1465-1466.

[7] Sprott, J.C., Some simple chaotic flows, Phys. Rev. E, 1994, 50(2), R647.

[8] Jafari S., Sprott J., Golpayegani S.M.R.H., Elementary quadratic chaotic flows with no equilibria, Phys. Lett. A, 2013, 377(9), 699702.

[9] Wang X., Pham V.-T., Jafari S., Volos C., Munoz-Pacheco J.M., Tlelo-Cuautle E., A new chaotic system with stable equilibrium: From theoretical model to circuit implementation, IEEE Access, 2017, 5, 8851-8858.

[10] Jafari S., Sprott J., Simple chaotic flows with a line equilibrium, Chaos, Solitons \& Fractals, 2013, 57, 79-84.

[11] Jafari S., Sprott J., Erratum to:"Simple chaotic flows with a line equilibrium"[Chaos, Solitons and Fractals 57 (2013) 79-84], Chaos Solitons \& Fractals, 2015, 77, 341-342.

[12] Pham V.-T., Jafari S., Volos C., Vaidyanathan S., Kapitaniak T., A chaotic system with infinite equilibria located on a piecewise linear curve, Optik, 2016, 127(20), 9111-9117.

[13] Barati K., Jafari S., Sprott J.C., Pham V.-T., Simple chaotic flows with a curve of equilibria, Int. J. Bifurcat. Chaos, 2016, 26(12), 1630034.

[14] Jafari S., Sprott J.C., Molaie M., A simple chaotic flow with a plane of equilibria, Int. J. Bifurcat. Chaos 26(06), 1650098 (2016).

[15] Sharma P.R., Shrimali M.D., Prasad A., Kuznetsov N., Leonov G., Controlling dynamics of hidden attractors, Int. J. Bifurcat. Chaos, 2015, 25(04), 1550061.

[16] Danca M.-F., Kuznetsov N., Hidden chaotic sets in a Hopfield neural system, Chaos, Solitons \& Fractals, 2017, 103, 144-150.

[17] Danca M.-F., Kuznetsov N., Chen G.: Unusual dynamics and hidden attractors of the Rabinovich-Fabrikant system, Nonlinear Dyn., 2017, 88(1), 791-805.

[18] Kuznetsov N., Leonov G., Yuldashev M., Yuldashev R., Hidden attractors in dynamical models of phase-locked loop circuits: limitations of simulation in MATLAB and SPICE, Commun. Nonlinear Sci. Numer. Simul., 2017, 51, 39-49.

[19] Leonov G., Kuznetsov N., Mokaev T., Hidden attractor and homoclinic orbit in Lorenz-like system describing convective fluid motion in rotating cavity, Commun. Nonlinear Sci. Numer. Simul., 2015, 28(1), 166-174.

[20] Leonov G.A., Kuznetsov N.V., Mokaev T.N., Homoclinic orbits, and self-excited and hidden attractors in a Lorenz-like system describing convective fluid motion, Eur. Phys. J. Spec. Top., 2015, 224(8), 1421-1458.

[21] Sharma P., Shrimali M., Prasad A., Kuznetsov N., Leonov G., Control of multistability in hidden attractors, Eur. Phys. J. Spec. Top., 2015, 224(8), 1485-1491.

[22] Kengne J., Njitacke Z., Fotsin H., Dynamical analysis of a simple autonomous jerk system with multiple attractors, Nonlinear Dyn., 2016, 83(1-2), 751-765.

[23] Kengne J., Njitacke Z., Nguomkam Negou A., Fouodji Tsostop M., Fotsin, H., Coexistence of multiple attractors and crisis Route to chaos in a novel chaotic jerk circuit, Int. J. Bifurcat. Chaos, 2016, 26(05), 1650081.

[24] Kengne J., Tabekoueng Z.N., Fotsin H., Coexistence of multiple attractors and crisis route to chaos in autonomous third order Duffing-Holmes type chaotic oscillators, Commun. Nonlinear Sci. Numer. Simul., 2016, 36, 29-44.

[25] Kengne J., Negou A.N., Tchiotsop D., Antimonotonicity, chaos and multiple attractors in a novel autonomous memristor-based jerk circuit, Nonlinear Dyn., 2017, 88(4), 2589-2608 (2017).

[26] Fouda J.A.E., Koepf W., Jacquir S., The ordinal Kolmogorov-Sinai entropy: A generalized approximation, Commun. Nonlinear Sci. Numer. Simul., 2017, 46, 103-115.

[27] Baptista M., Ngamga E., Pinto P.R., Brito M., Kurths J., Kolmogorov-Sinai entropy from recurrence times, Phys. Lett. A, 2010, 374(9), 1135-1140.

[28] Kapitaniak T., Leonov G.A., Multistability: uncovering hidden attractors, Eur. Phys. J. Spec. Top., 2015, 224(8), 1405-1408.

[29] Jaros P., Kapitaniak T., Perlikowski P., Multistability in nonlinearly coupled ring of Duffing systems, Eur. Phys. J. Spec. Top., 2016, 225(13-14), 2623-2634.

[30] Chudzik A., Perlikowski P., Stefanski A., Kapitaniak T., Multistability and rare attractors in van der Pol-Duffing oscillator. Int. J. Bifurcat. Chaos, 2011, 21(07), 1907-1912.

[31] Lai Q., Chen S., Research on a new 3D autonomous chaotic system with coexisting attractors, Optik, 2016, 127(5), 3000-3004. 
[32] Lai Q., Akgul A., Zhao X.-W., Pei H., Various types of coexisting attractors in a new $4 \mathrm{D}$ autonomous chaotic system. Int. J. Bifurcat. Chaos, 2017, 27(09), 1750142.

[33] Pan W., Li L., Degenerate Hopf bifurcation in a self-exciting Faraday disc dynamo, Pramana, 2017, 88(6), 87.

[34] Pisarchik A.N., Feudel U., Control of multistability. Phys. Rep., 2014, 540(4), 167-218.

[35] Ahn C.K., T-S fuzzy Ho synchronization for chaotic systems via delayed output feedback control. Nonlinear Dyn., 2010, 59(4), 535-543.

[36] Ahn C.K., Chaos synchronization of nonlinear Bloch equations based on input-to-state stable control, Commun. Theor. Phys., 2010, 53(2), 308.

[37] Ahn C.K., Output feedback $\mathrm{H} \infty$ synchronization for delayed chaotic neural networks, Nonlinear Dyn., 2010, 59(1-2), 319.

[38] Ahn C.K., Neural network $\mathrm{H} \infty$ chaos synchronization, Nonlinear Dyn., 2010, 60(3), 295-302.

[39] Ahn C.K., Robust chaos synchronization using input-to-state stable control, Pramana, 2010, 74(5), 705-718.

[40] Ahn C.K., Jung S.-T., Kang S.-K., Joo S.-C., Adaptive $\mathrm{H} \infty$ synchronization for uncertain chaotic systems with external disturbance, Commun. Nonlinear Sci. Numer. Simul., 2010, 15(8), 2168-2177.

[41] Ahn C.K., Kim P.S., T-S fuzzy adaptive delayed feedback synchronization for time-delayed chaotic systems with uncertain parameters, Int. J. Mod. Phys. B., 2011, 25(23n24), 3253-3267.
[42] Ahn C.K., Takagi-Sugeno fuzzy receding horizon $\mathrm{H} \infty$ chaotic synchronization and its application to the Lorenz system, Nonlinear Anal. Hybrid Syst., 2013, 9, 1-8.

[43] Ahn C.K., An $\mathrm{H} \infty$ approach to anti-synchronization for chaotic systems, Phys. Lett. A, 2009, 373(20), 1729-1733.

[44] Volos C.K., Kyprianidis I.M., Stouboulos I.N., Image encryption process based on chaotic synchronization phenomena, Signal Process., 2013, 93(5), 1328-1340.

[45] Volos C., Akgul A., Pham V.-T., Stouboulos I., Kyprianidis I., A simple chaotic circuit with a hyperbolic sine function and its use in a sound encryption scheme, Nonlinear Dyn., 2017, 89(2), 1047-1061.

[46] Molaie M., Jafari S., Sprott J.C., Golpayegani S.M.R.H., Simple chaotic flows with one stable equilibrium, Int. J. Bifurcat. Chaos, 2013, 23(11), 1350188.

[47] Sprott J.C., Elegant chaos: algebraically simple chaotic flows, World Scientific, Singapore, 2010.

[48] Cover T.M., Thomas J.A., Elements of information theory, 2nd ed., John Wiley \& Sons, New Jersey, 2006.

[49] Shannon C.E., Weaver W., The mathematical theory of communication, University of Illinois Press, USA, 1998.

[50] Kolmogorov A.N., Entropy per unit time as a metric invariant of automorphisms, Dokl. Akad. Nauk SSSR, 1959, 4, 754-755.

[51] Young L.-S., What are SRB measures, and which dynamical systems have them?, J. Stat. Phys., 2002, 108(5), 733-754.

[52] Pesin Y.B., Characteristic Lyapunov exponents and smooth ergodic theory, Russ. Math. Surv. 1977, 32(4), 55-114. 